\title{
Platelet Sequestration in Man. II. Immunological and Clinical Studies *
}

\author{
Richard H. Aster † and James H. Jandl \\ (From the Thorndike Memorial Laboratory and Second and Fourth [Harvard] Medical \\ Services, Boston City Hospital, and the Department of Medicine, Harvard \\ Medical School, Boston, Mass.)
}

The mechanisms of platelet destruction and their sites of sequestration in disease states are poorly understood. Although recent studies have emphasized the importance of platelet isoantibodies in certain clinical situations (1-3), little is known of the effects of antibodies on platelets in vivo. In conditions such as idiopathic thrombocytopenic purpura, there is debate as to whether immune mechanisms are involved, and sites of platelet sequestration are unknown.

The availability of a method for labeling platelets with $\mathrm{Cr}^{51}$ that allows most of the transfused platelets to remain viable and reduces the initial deposition of nonviable platelets in the liver and spleen (4) permits study of some of these aspects of platelet pathophysiology. We investigated the survival and sites of sequestration of platelets exposed to isoantibodies in vivo. Included in this paper are observations of pyrogenic reactions after immune platelet destruction. Studies in patients with idiopathic thrombocytopenic purpura and other platelet disorders are also described.

\section{Methods}

Preparation and transfusion of $\mathrm{Cr}^{51}$-labeled "citrate platelets" and determination of the percentage of platelet survival were as described in the preceding report (4). In "extracting" $\mathrm{Cr}^{51}$-labeled platelets from blood samples taken from thrombocytopenic persons, nonradioactive platelets were added to the platelet-rich plasma to facilitate recovery of platelet $\mathrm{Cr}^{51}$. For isologous transfusions, only platelets from donors compatible with the recipients' $\mathrm{ABO}$ and $\mathrm{Rh}$ blood types were used.

Methods of scintillation scanning over organs to evaluate sites of platelet sequestration have been described

* Submitted for publication October 1, 1963; accepted January 2, 1964.

Supported in part by grant HE-07652-01 from the National Institutes of Health.

$\dagger$ Postdoctoral fellow, National Institutes of Health, HPD-18,183-02.
$(4,5)$. Units employed to measure organ radioactivity were derived by dividing the counts per minute observed over an organ by 10 times the number of microcuries of platelet $\mathrm{Cr}^{51}$ injected. This eliminated variations caused solely by the specific radioactivity of the transfused platelets.

Platelet counts were done with phase microscopy (6) using duplicate pipettes. When platelet levels were less than 50,000 per $\mathrm{mm}^{3}$, a dilution of $1: 20$, rather than $1: 100$, was used, and additional chambers were counted to increase accuracy.

Subjects selected for study were patients on the Medical Services of the Boston City Hospital. Those designated as "normal" had minor disorders unrelated to the blood or blood-forming organs. None had palpable enlargement of the liver or spleen or showed abnormality in hepatic function tests.

\section{Results}

Sequestration of platelets in the isoimmune state. After repeated transfusion of platelets refractoriness may develop in the recipient so that the $\mathrm{Cr}^{51}$ survival of subsequently transfused platelets is decreased (7-9). Two patients were studied who showed this phenomenon in different degrees.

The first patient had received from a single donor two previous platelet transfusions that survived normally. The survival curve that resulted from the third transfusion, using platelets from the same donor, is given in Figure 1. The transfused platelets disappeared from the circulation over a 48-hour period, and there was a corresponding increase in radioactivity levels over the spleen but no change over the liver or lung. The half-disappearance time ( $\left.t \frac{1}{2}\right)$ of transfused platelets was about 19 hours.

The second patient had received 5 previous platelet transfusions. Only the first 3 had been labeled with $\mathrm{Cr}^{51}$, and those showed normal survivals. After the sixth transfusion, platelets disappeared rapidly from the blood stream, and there 


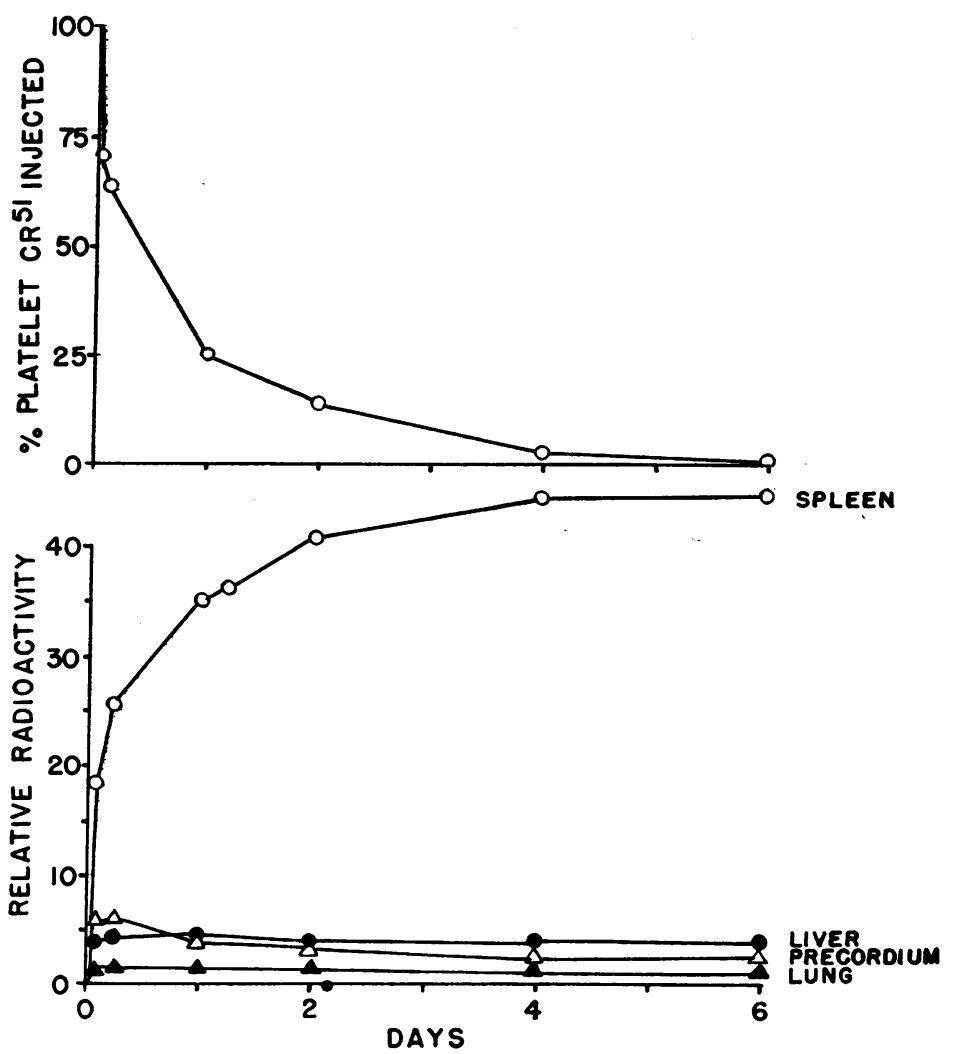

Fig. 1. Sequestration of isologous "citrate platelets" transfused TO A PATIENT IN THE EARLY STAGE OF ISOIMMUNITY.

was a corresponding and approximately equal increase in radioactivity over both the liver and the spleen (Figure 2). These values indicate that about $80 \%$ of the platelet $\mathrm{Cr}^{51}$ was sequestered in the liver (see below). The half-disappearance time of these platelets was less than 30 minutes. Interestingly, a small fraction of the transfused platelets remained in the circulation, gradually disappearing over a 9-day period.

Serologic studies using these patients' sera and the same platelets that were transfused were negative for antibody using agglutination and complement $\left(\mathrm{C}^{\prime}\right)$-fixation techniques $(2,10)$.

Sequestration of platelets after reaction with $a$ specific $C^{\prime}$-fixing isoantibody. Although the destruction of platelets in the above patients was probably a manifestation of isoimmunity, no antibodies could be demonstrated by in vitro testing. It was of interest, therefore, to study the pattern of sequestration resulting when transfused platelets reacted with an isoantibody of known potency and specificity. For this purpose the antibody used was one produced by a patient who had been repeatedly transfused for treatment of thrombocytopenia (3). Serum from this patient fixed $C^{\prime}$ in a final dilution of $1: 300$ with appropriate platelets, but the antibody could not be detected by other conventional serologic techniques (10). It reacted with an antigen, first called $\mathrm{Pl}^{\mathrm{B} 1}$ (11), which is present on platelets of about $48 \%$ of normal persons (10). This antigen can also be demonstrated on leukocytes by appropriate absorption and $\mathrm{C}^{\prime}$-fixation techniques $(2,12)$.

The effect of this antibody on the survival of platelets containing the homologous antigen was studied by passive immunization techniques. $\mathrm{Cr}^{51}$-labeled platelets containing the $\mathrm{Pl}^{\mathrm{B} 1}$ antigen were transfused into four normal subjects who lacked that antigen, and the platelet $\mathrm{Cr}^{51}$ activity was followed for 1 or 2 days to assure that the survival pattern was normal. Different amounts of serum containing the antibody (which reacted 
in vitro with the donor's platelets but not with those of the recipient) were then injected intravenously. Circulating platelet $\mathrm{Cr}^{51}$ activity, total platelet count, total differential leukocyte counts, body surface radioactivity, and the subject's symptoms and body temperature were then followed for a 24-hour period.

Changes in circulating platelet $\mathrm{Cr}^{51}$ activity and body surface radioactivity are summarized in Figures 3 and 4 . After injection of $0.25 \mathrm{ml}$ of this antibody, $\mathrm{Cr}^{51}$-labeled platelets disappeared from the circulation with a $t_{2} \frac{1}{2}$ of 160 minutes; there was a corresponding reciprocal increase in radioactivity over the spleen. No change in hepatic radioactivity occurred (Figure 3 ). After injection of larger amounts of antibody (2.0 or $2.5 \mathrm{ml}$ ), labeled platelets disappeared more rapidly from the blood stream ( $t_{2} \frac{1}{2}=15$ minutes), and the increase in organ radioactivity was chiefly hepatic (Figure 4). With an intermediate amount of antibody $(0.5 \mathrm{ml})$ the $t_{\frac{1}{2}}$ was 80 minutes, and an increase in $\mathrm{Cr}^{51}$ activity occurred in both liver and spleen but chiefly over the latter organ (Figure 3 ). In no instance was an increase in radioactivity observed over the lungs.

The changes in other blood elements accompanying the sequestration of $\mathrm{Cr}^{51}$-labeled platelets are given in Figure 5. In each experiment, injection of antibody was followed by a variable degree of leukopenia characterized by a reduction in neutrophiles, monocytes, and eosinophiles, which lasted for several hours. As the leukocyte count returned to normal levels, "band" forms appeared, constituting up to $30 \%$ of the granulocytes. In one subject (No. 2 ), a striking leukocytosis followed. In three subjects (No. 1, 3, and 4), injection of antibody was followed by a significant reduction in total platelet levels.

Three of the four subjects (No. 1, 3, and 4) developed pyrogenic reactions characterized as follows: No symptoms were observed for 30 minutes; then frontal headache developed, lasting from 1 to 2 hours. At 45 to 50 minutes, a frank, shaking chill occurred, lasting from 15 to $30 \mathrm{~min}-$
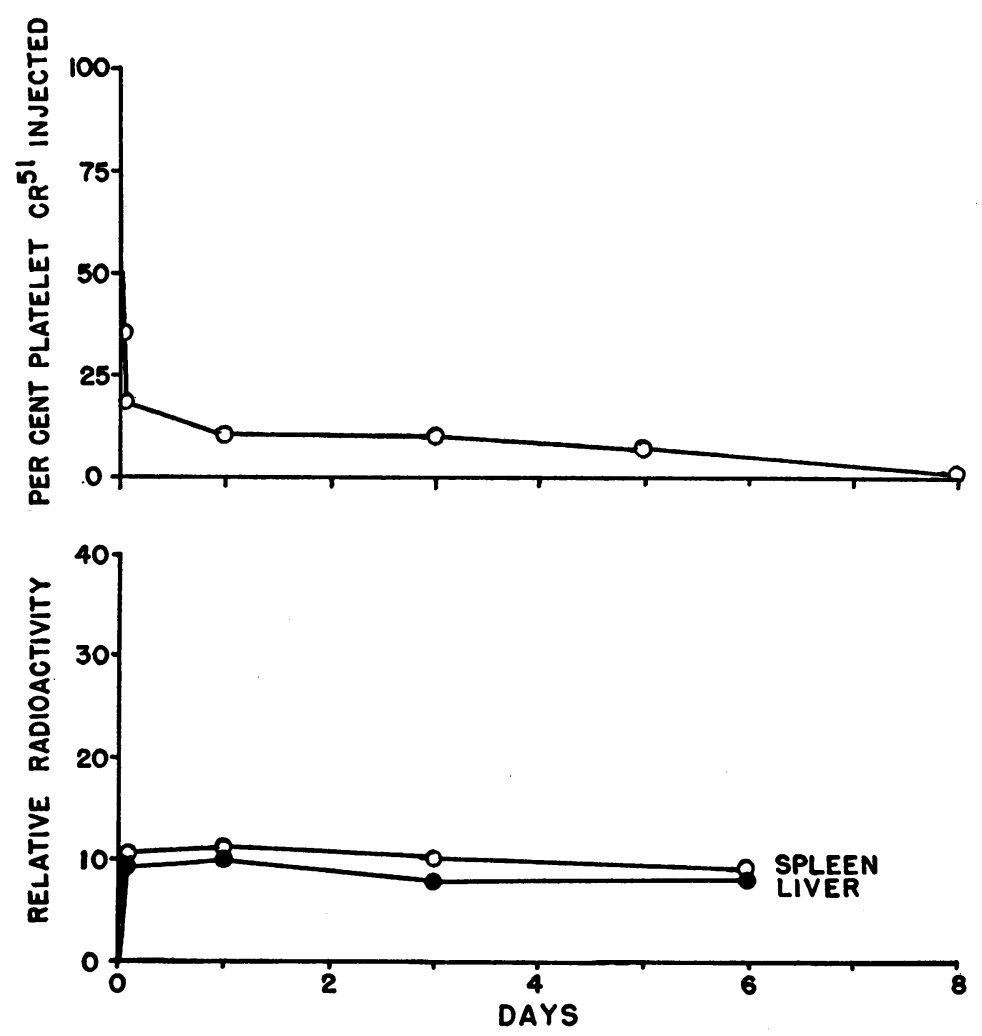

Fig. 2. Sequestration of isologous "Citrate platelets" transfused to A PATIENT With LATE isoim MUNity. 

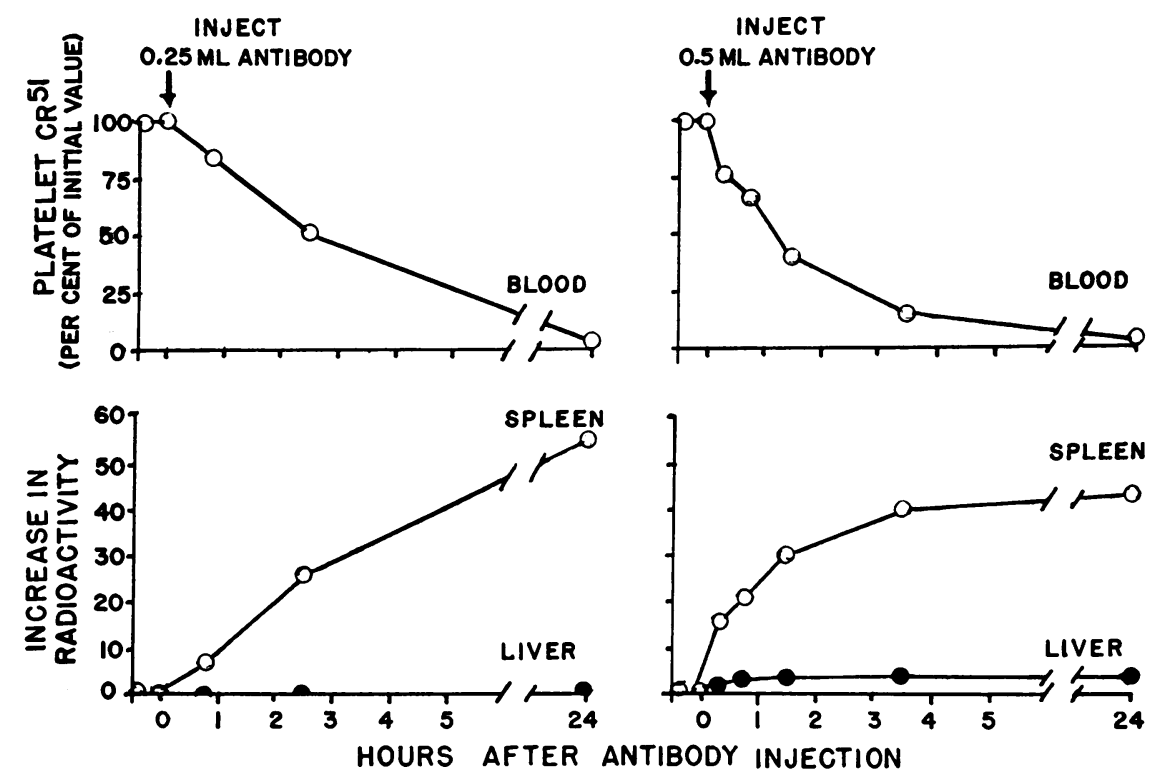

Fig. 3. Sequestration of PReviously transfused $C_{R^{51}}$-Labeled platelets in SubJECTS AFTER INJECTION OF RELATIVELY SMALL AMOUNTS OF ISOANTIBODY.

utes. As the chill subsided, fever appeared that persisted for 1 to 4 hours. In one subject (No. 2 ), a headache that appeared about 40 minutes after antibody injection was the only symptom. Characteristics of these reactions and their relationship to antibody:antigen ratio and the rate and site of platelet sequestration are given in Figure 6.
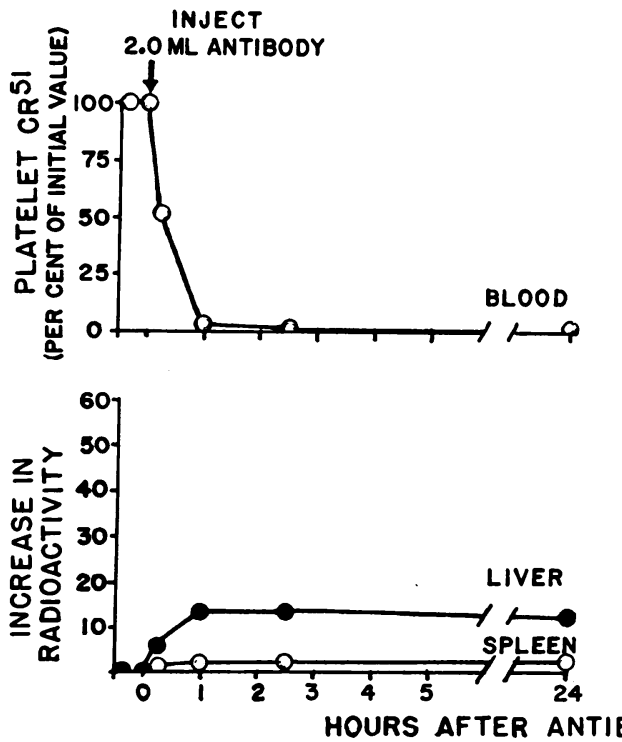

When comparable amounts of antibody were injected into subjects who had no circulating $\mathrm{Pl}^{\mathrm{B1}}$ antigen, no reactions or changes in blood elements occurred. The same was true when antibody was injected into $\mathrm{Pl}^{\mathrm{B} 1}$-positive subjects, thus producing a very low antibody: antigen ratio.

Idiopathic thrombocytopenic purpura (ITP). Chronic idiopathic thrombocytopenic purpura. A
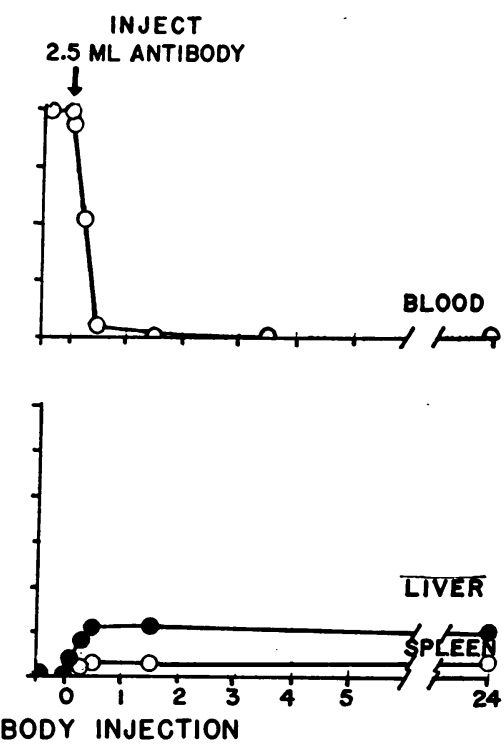

Fig. 4. Sequestration of PReviously transfused $C^{51}$-Labeled platelets in subJECTS AFTER INJECTION OF RELATIVELY LARGE AMOUNTS OF ISOANTIBODY. 
1.

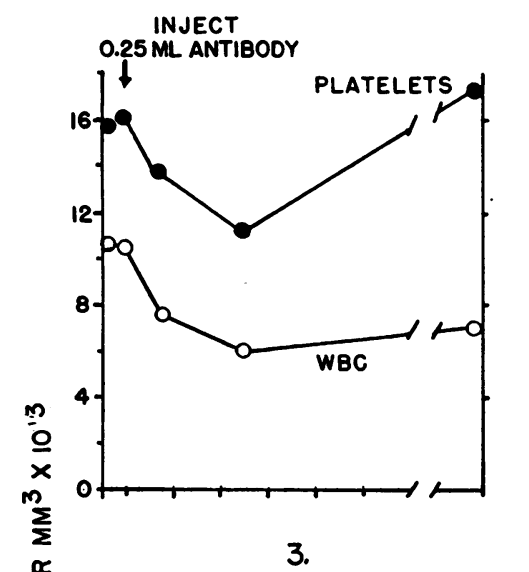

2.
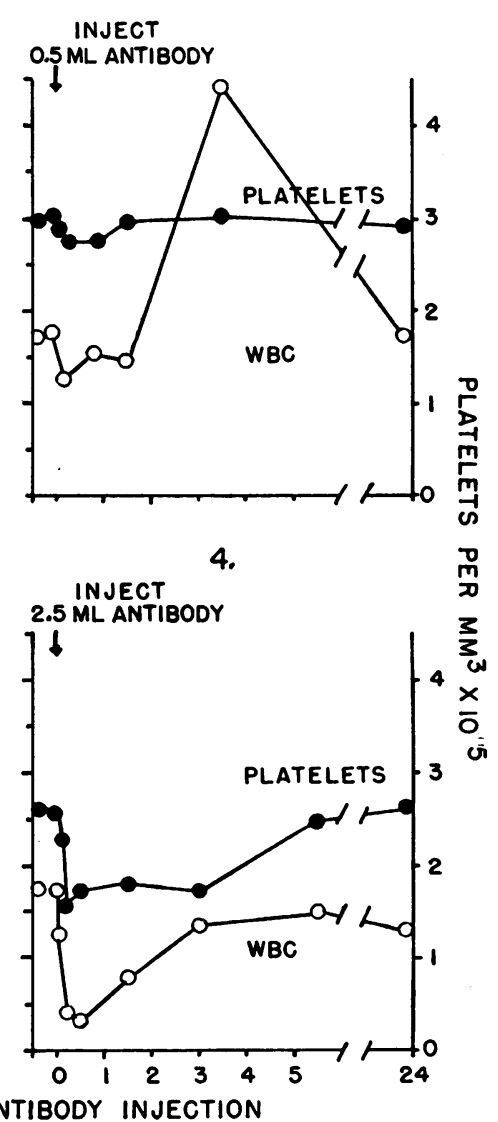

Fig. 5. Changes in total leukocyte and Platelet levels after REACTION OF TRANSFUSED $C_{R^{51}}$-LABELED PLATELETS WITH INJECTED ISOANTIBODY IN FOUR NORMAL SUBJECTS. (See Figures 3 and 4 ). WBC $=$ white blood cells.

68-year-old diabetic female was found to have purpura and a platelet count of 15,000 per $\mathrm{mm}^{3}$. All findings were consistent with chronic idiopathic thrombocytopenic purpura. Because steroid hormones affected her diabetic control, she had been followed without this medication for 6 months without external bleeding or recurrences of purpura. She had never received a blood transfusion. When $\mathrm{Cr}^{51}$-labeled, isologous, $\mathrm{ABO}$ compatible platelets were given, the usual sudden loss of nonviable platelets occurred (4), followed by a slower removal of $\mathrm{Cr}^{51}$ platelets from the circulation with a half-disappearance time of 7 hours (Figure 7). During this time, there was a progressive increase in splenic radioactivity and no change over the liver. The patient was then given prednisone, $40 \mathrm{mg}$ daily, for 5 days, which caused the platelet level to increase to 60,000 per $\mathrm{mm}^{3}$. At this point, again using isologous platelets, a second survival study was carried out. The half-disappearance time had increased to about 14 hours. Again, splenic sequestration was observed. When the two curves were plotted with semilogarithmic coordinates, the disappearance of $\mathrm{Cr}^{51}$-labeled platelets was found to be first order with time after completion of the initial rapid destruction of platelets rendered nonviable by the labeling procedure.

A 52-year-old man with chronic alcoholism and known idiopathic thrombocytopenic purpura of 2 years' duration was admitted when he stopped taking prednisone and developed purpura. Platelets were 24,000 per $\mathrm{mm}^{3}$. He had previously received approximately six blood transfusions during operative procedures. An isologous platelet survival study was begun before steroid hor- 


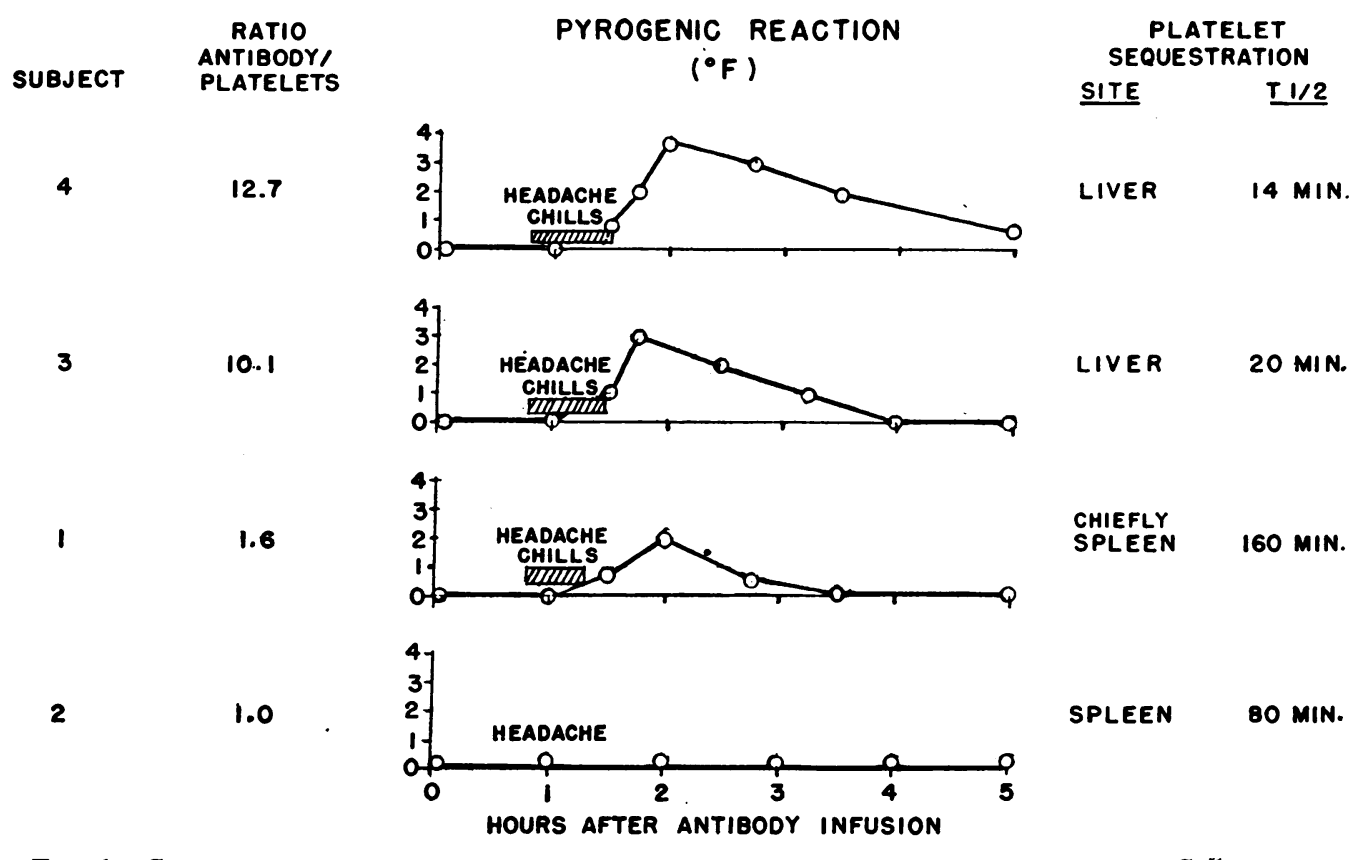

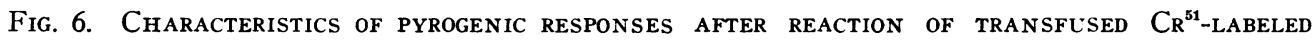
PLATELETS WITH INJECTED ISOANTIBODY IN FOUR NORMAL SUBJECTS. Ratio of antibody: platelets is defined as milliliters of antibody-containing serum injected per $10^{11}$ (approximately $1 \mathrm{ml}$ ) $\mathrm{Pl}^{\mathrm{B1}}$-positive platelets circulating in the recipient.

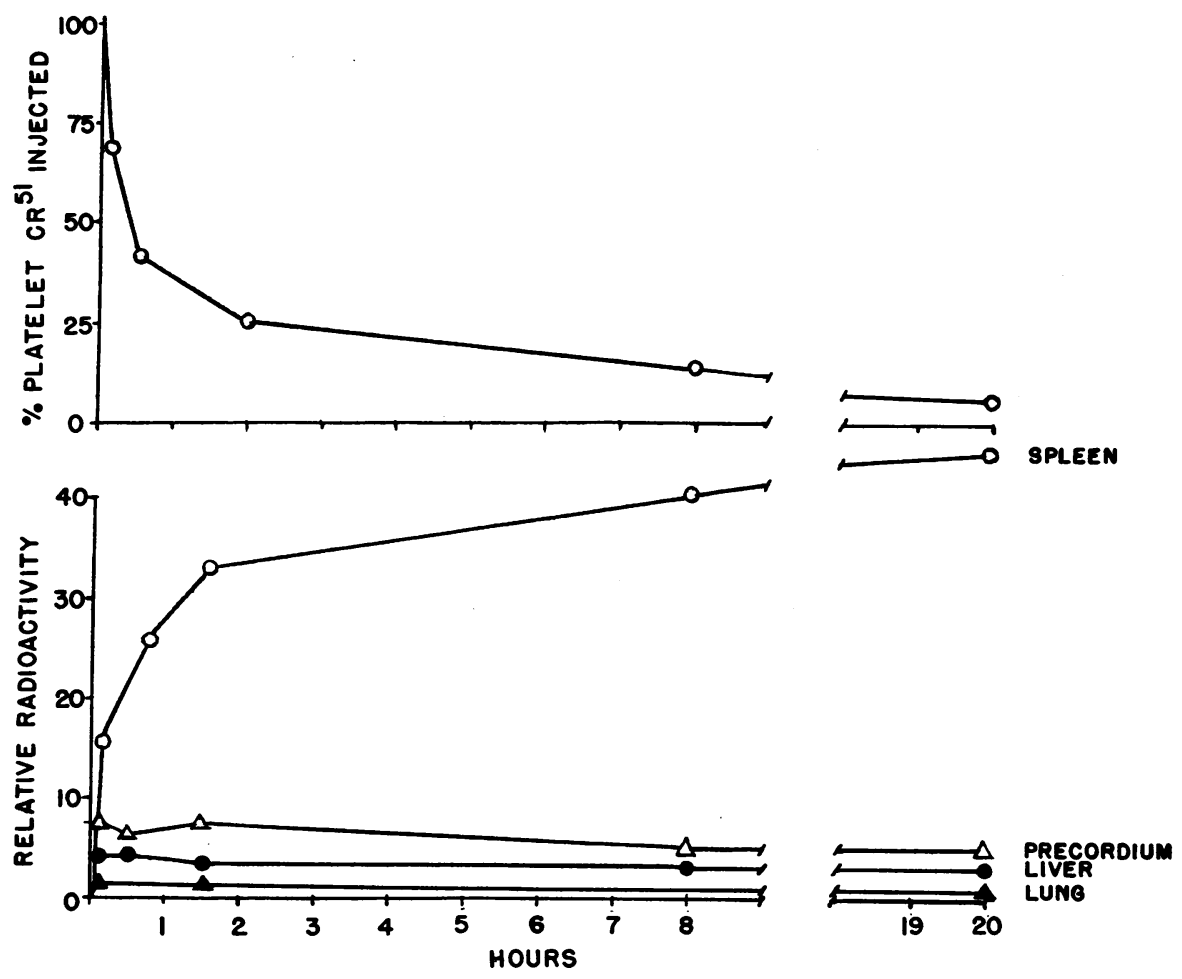

Fig. 7. Sequestration of isologous $C_{R}{ }^{51}$-labeled platelets in a patient with CHRONIC IDIOPATHIC THROMBOCYTOPENIC PURPURA (ITP) BEFORE CORTICOSTEROID THERAPY, 
mone therapy was instituted. Only $50 \%$ of the transfused platelets were recovered, but the lifespan of surviving platelets was entirely normal, and no unusual changes in organ radioactivity occurred. A rapid rise in platelet levels to normal resulted when prednisone therapy was begun.

Acute idiopathic thrombocytopenic purpura. A 64-year-old man, previously well, experienced the sudden onset of purpura and bleeding from the gums and gastrointestinal and urinary tracts. Platelets were 3,000 per $\mathrm{mm}^{3}$; megakaryocytes were abundant in the bone marrow. The patient had never been transfused. Although the clinical picture resembled that occurring with haptenedependent platelet antibodies of the quinidine type, no history of exposure to drugs or toxins could be elicited, and tests with the patient's serum were negative for platelet antibodies in both agglutination and complement-fixation techniques.
At the time prednisone therapy was begun, an isologous platelet survival study was carried out. Transfused platelets disappeared rapidly ( $t \frac{1}{2}$ about 15 minutes) with a corresponding rise in radioactivity levels of approximately equal magnitude over the liver and spleen (Figure 8). These values suggest that about $80 \%$ of the transfused platelets were destroyed in the liver (see Discussion).

After 10 days of prednisone therapy, there was a gradual rise in platelet levels to normal, which persisted after the medication was discontinued. Platelet survival was normal after remission occurred.

Congestive splenomegaly with thrombocytopenia. Studies were made in two patients with congestive splenomegaly secondary to portal cirrhosis who had platelet levels of 50,000 and 90,000 per $\mathrm{mm}^{3}$, respectively. In each case, survival of
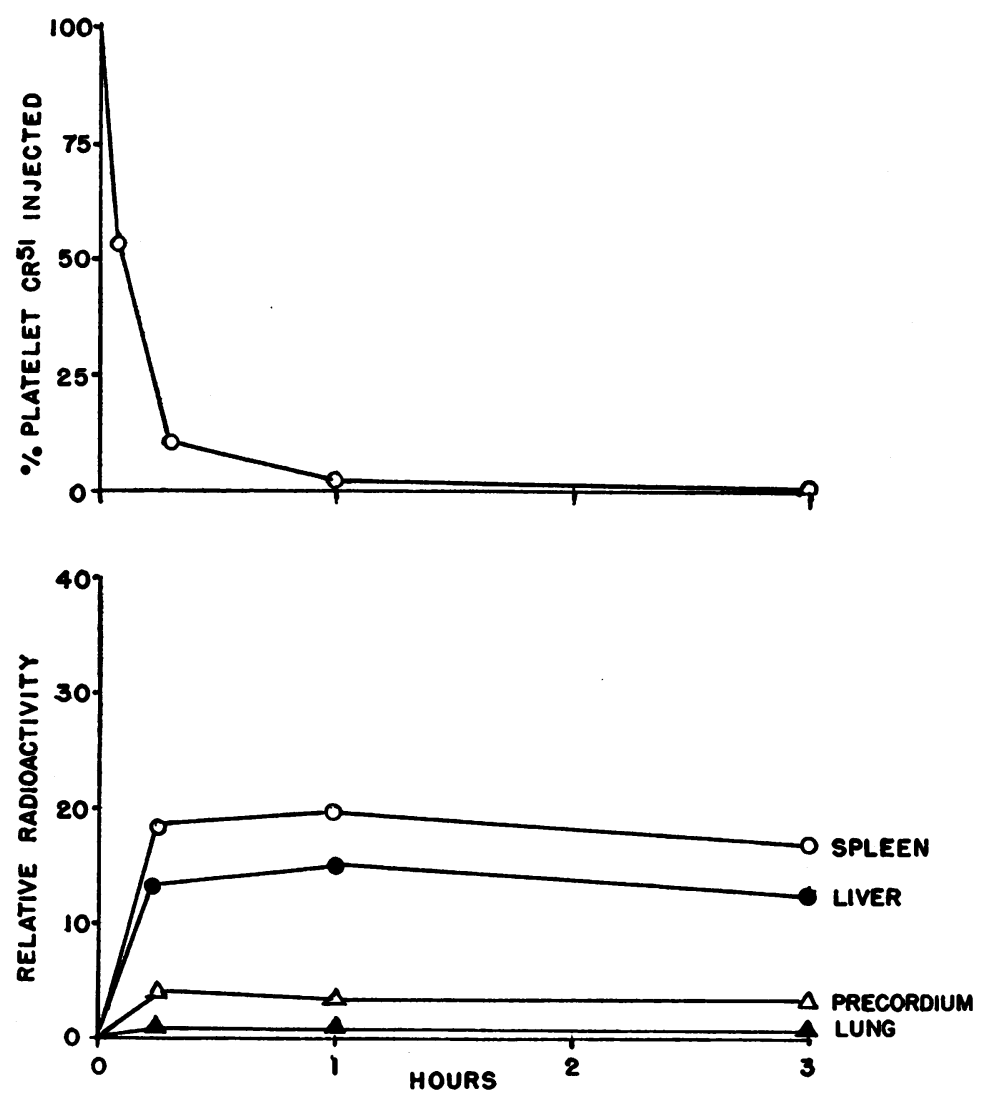

Fig. 8. Sequestration of isologous "citrate platelets" in a patient with acute ITP and a platelet count of 3,000 per cubic MILLIMETER, 


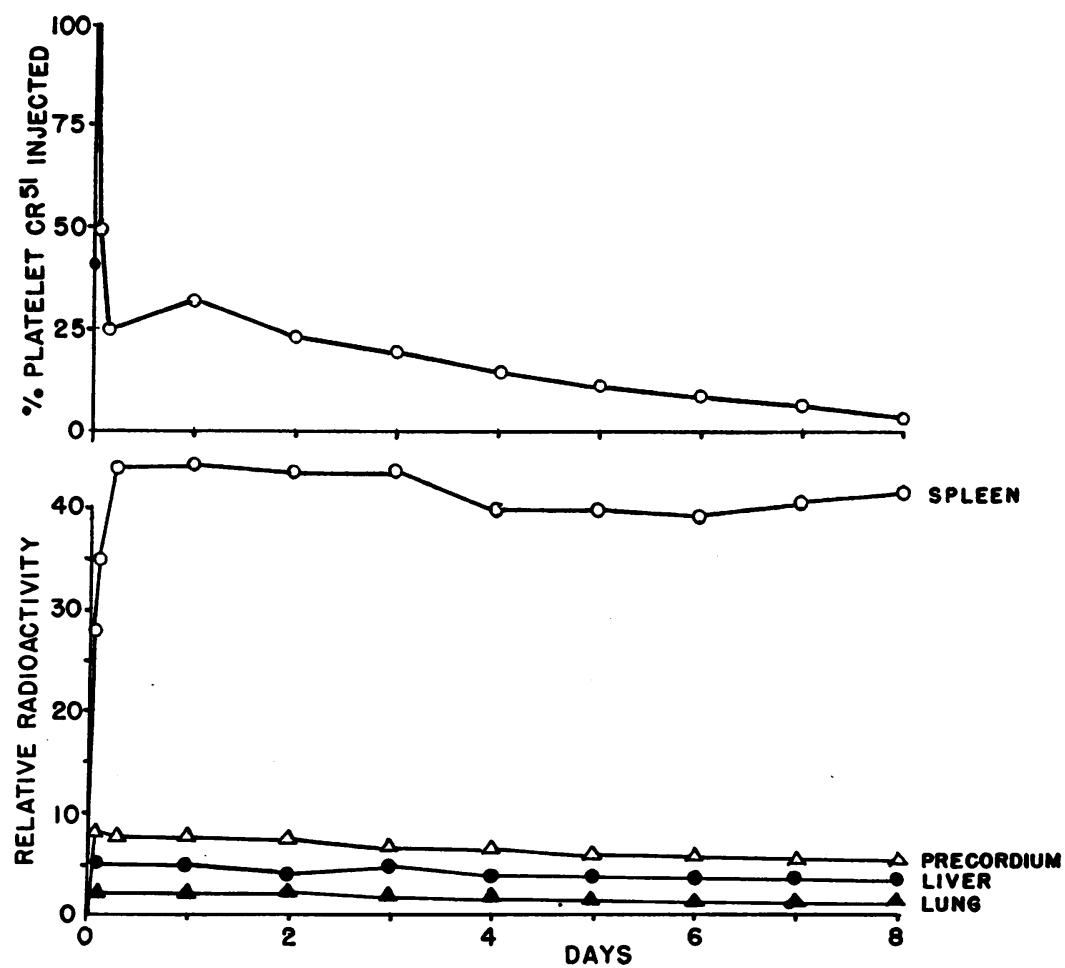

Fig. 9. Sequestration of autogenous "citrate platelets" in congesTIVE SPLENOMEgaly With thrombocytopenia. (Platelets, 90,000 per $\mathrm{mm}^{3}$.) There is an extraordinary initial splenic uptake of injected platelets.

autologous platelets was well within normal limits although the recovery of the transfused platelets was low ( $30 \%$ and $50 \%$, respectively). Organ scanning was done in only one subject, who showed a marked initial splenic uptake (Figure 9). No further splenic or hepatic accumulation of $\mathrm{Cr}^{51}$ occurred during an 8-day period of observation, nor was there the expected decline in organ radioactivity.

Idiopathic thrombocythemia. A 72-year-old woman with no symptoms relating to hematologic disease was found to have a mild anemia and platelet level of $1,500,000$ per $\mathrm{mm}^{3}$. The bone marrow was normal, and a diagnosis of idiopathic thrombocythemia was made. Eighty-two per cent of autologous transfused platelets were viable, but the disappearance curve deviated from a straight line, suggesting some superimposed random destruction of platelets (Figure 10). No unusual accumulation of radioactivity in liver, spleen, or lungs was observed.

Relationship between surface radioactivity and total organ radioactivity. In experiments where known amounts of radioactivity were selectively deposited either in the liver or the spleen, it was possible by a standard scanning technique (4) to correlate the known increase in organ $\mathrm{Cr}^{51}$ with the increase observed over the surface. As shown in Figure 11, for both organs a relationship approximating a straight line existed over a wide range of radioactivity in different subjects. One $\mu \mathrm{c}$ of $\mathrm{Cr}^{51}$ in the spleen was found to produce approximately $512 \mathrm{cpm}$ at the surface, whereas 1 $\mu \mathrm{c}$ in the liver gave $132 \mathrm{cpm}$. Radioactivity in the spleen was thus detected about 4 times as effectively as in a larger organ, the liver. As emphasized previously (4), such relationships would not be expected to hold in subjects with enlarged organs or extremes of body habitus. Furthermore, the assumption that all, or nearly all, the sequestered platelets went to the organ under consideration could be proved only by removal of the organ and direct $\mathrm{Cr}^{51}$ assay. However, since a significant increase in radioactivity occurred over 

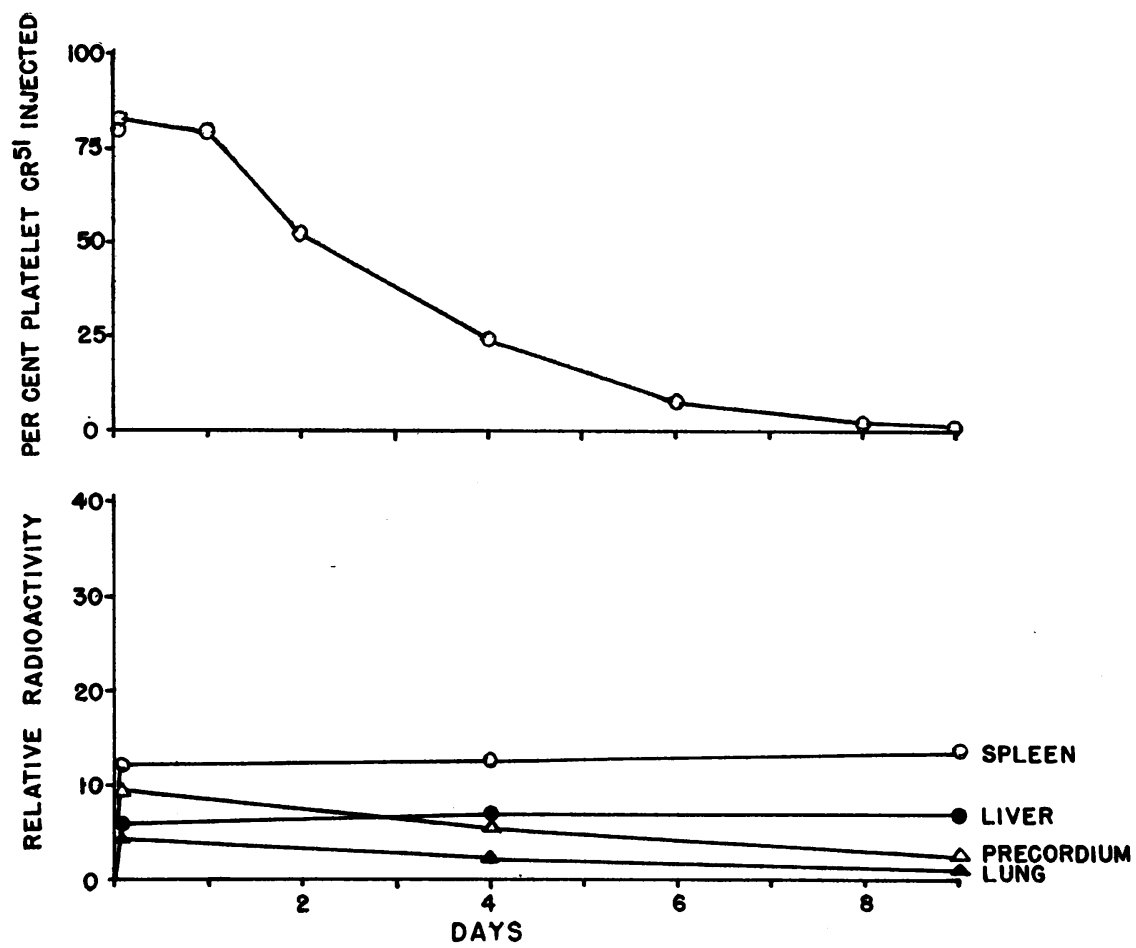

Fig. 10. Sequestration of autogenous “citrate platelets" in a patient with idiopathic THROMBOCYTHEMia. (Platelets, $1,800,000$ per $\mathrm{mm}^{3}$.)

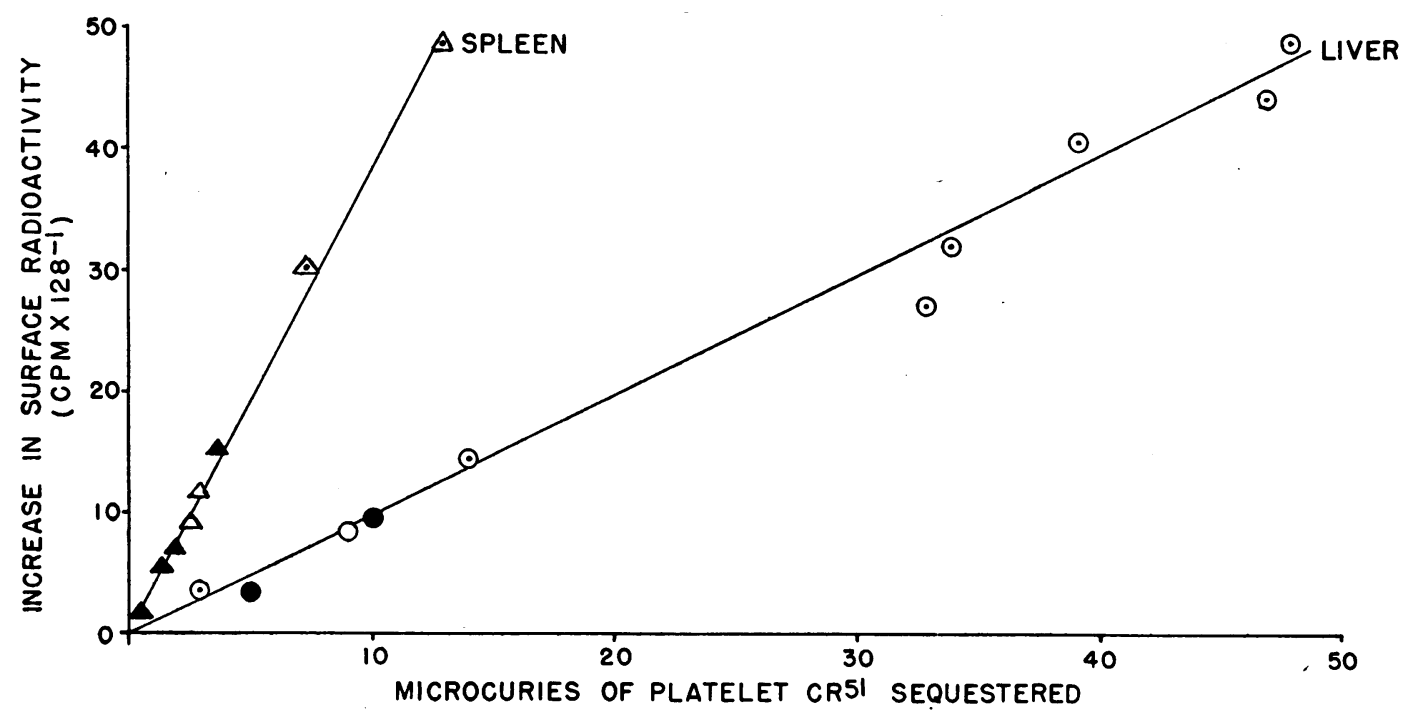

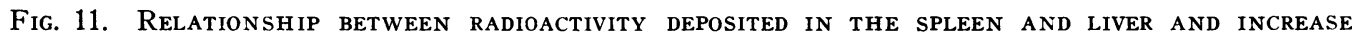
IN SURface COUNTS PER MINUTE. Experiments are those in which a significant increase in surface radioactivity occurred over only one organ. Splenic curve : $\boldsymbol{\Delta}=$ reaction of platelets with small amounts of isoantibody, $\Delta=$ chronic ITP, $\Delta=$ early isoimmunity. Hepatic curve: $\bullet=$ reaction of platelets with large amounts of isoantibody, $\mathrm{O}=$ acute ITP, $\odot=$ "EDTA platelets" 30 minutes after transfusion (4). 
only one site in the studies selected and there was no associated increase in urinary $\mathrm{Cr}^{51}$ excretion, this assumption is probably justified.

That calculations based on the relationships shown in Figure 11 may be useful is shown in Subject 2 (Figure 3), where an intermediate amount of isoantibody caused platelets to be destroyed in both the spleen and liver. The increase in surface cpm over the spleen corresponded to $11.4 \mu \mathrm{c}$ of $\mathrm{Cr}^{51}$, whereas the rise over the liver represented $3.8 \mu \mathrm{c}$. The sum, $15.2 \mu \mathrm{c}$, was a good approximation to the total amount of $\mathrm{Cr}^{51}$ (13.2 $\mu \mathrm{c})$ known to have been sequestered in this subject.

\section{Discussion}

Our studies indicate that the transfusion of $\mathrm{Cr}^{51}$-labeled "citrate platelets" followed by body surface scanning may provide useful information concerning platelet behavior in a variety of pathologic states. More work will be required to fully explore the value of the technique, but certain conclusions can be drawn from the present data.

Antibody-induced platelet sequestration: pyrogenic reactions and associated hematologic changes. The pattern of organ sequestration after platelets were transfused to subjects in a state of isoimmunity and after transfused platelets reacted with injected isoantibody indicates that minimal sensitization of platelets with antibody induces a lesion perceived only by the spleen. With greater sensitization, platelets are destroyed chiefly in the liver. This rapid, hepatic sequestration can be caused by concentrations of antibody far too small to be detected by any of the usual serologic techniques. It is possible, of course, that platelet antibodies having other specificities would behave differently, but these findings parallel observations made with red cells sensitized with variable amounts of anti-D or anti-A antibodies $(13,14)$. The antiplatelet factor in the cases of idiopathic thrombocytopenic purpura studied apparently behaved like an antibody to the extent that in a mild form of the disease platelet sequestration was splenic, whereas in a severe form it was chiefly hepatic. Earlier studies of thrombocytopenia induced by ITP plasma [Harrington and coworkers (15)] are consistent with these observations. Nonimmunologic damage can, however, produce a similar pattern, since platelets injured by exposure to EDTA go almost exclusively to the liver but platelets altered by the milder acid-citrate anticoagulant are trapped in the spleen (4). The nature of the platelet lesion produced by these various means remains unclear. In vitro studies with the anti- $\mathrm{Pl}^{\mathrm{B} 1}$ antibody, serum from the patients with ITP, or serum obtained during isoimmunity, suggest that platelets were neither agglutinated nor lysed in vivo. If complement played any role, it did so in quantities too small to measure in vitro. That platelet destruction may be caused by concentrations of antibody in vivo that are not measurable in vitro has been reported by Shulman, Aster, Leitner, and Hiller (1) and is compatible with observations made on the immune destruction of red cells (13, 14).

Both the severity of the pyrogenic responses and the rate of platelet sequestration following the reaction of injected isoantibody and platelets in vivo were related to the ratio of antibody injected to platelet antigen circulating in the recipient. An exception was Subject 1 (Figure 3), where sequestration was slower than in Subject 2 , although the antibody: antigen ratio was higher in the first subject. Subject 1 had the smallest amounts of both antibody and antigen circulating, and at these low concentrations, the antigen-antibody combination may have been incomplete.

As with red cells (16) the induction of a pyrogenic reaction clearly depends upon more than the simple combination of platelet antigen and antibody in vivo. This is illustrated by experiments where antibody injected into persons, all of whose platelets contained the specific antigen, caused no symptoms at all and suggests that a certain minimal concentration of antibody on the surface of the cell is necessary to cause a pyrogenic response. It has been shown that the requirements for complement to be fixed are somewhat similar $(1,17,18)$, but the fact that anti-D, which fixes little or no $C^{\prime}$, is extremely pyrogenic upon combining with red cells (16) indicates that $\mathrm{C}^{\prime}$ fixation is probably not essential.

Since the platelet isoantibody used to induce platelet sequestration cross-reacted with leukocytes, the possibility must be considered that the pyrogenic responses observed were caused by the combination of antibody with the small numbers of leukocytes that contaminated the transfused plate- 
let preparations. The number of leukocytes transfused was calculated in two of the isoantibody experiments and found to be $5 \times 10^{6}$ and $4 \times 10^{7}$. This indicates the presence of only 1 leukocyte for every 10,000 and 1,700 platelets, respectively. The smallest number of leukocytes was given to Subject 4 , who had the most severe reaction, and the largest number was given to Subject 2, who had no fever or chills. It seems unlikely that the combination of isoantibodies with these small amounts of leukocytes could explain the symptoms observed.

The symptomatology of the pyrogenic reactions observed in Subjects 1, 3, and 4 was similar to that commonly occurring in the course of routine blood transfusion therapy (19). In the absence of any demonstrable red cell incompatibility, it is often assumed that such reactions are due to leukocyte antibodies, or "leukoagglutinins." One recent study (20), however, showed that febrile transfusion reactions do not correlate well with the presence of leukoagglutinins in the recipients' serum. Undoubtedly transfusion reactions can be caused by leukocyte antibodies. For experimental purposes, however, it is far easier to obtain platelets free of leukocytes than leukocytes free of platelets. The present study indicates that platelet-antiplatelet reactions can cause severe feverchill responses and suggests that platelet antigens may be an important cause of transfusion reactions observed in clinical medicine.

The leukopenia that followed the platelet antigen-antibody reaction has been shown to be characteristic of many types of in vivo immune reactions $(16,21,22)$. In the four patients studied, the rate and magnitude of the reduction in the leukocyte count was roughly proportional to the rate at which $\mathrm{Cr}^{51}$-labeled platelets were destroyed, suggesting that leukocytes were directly affected by sensitized platelets. Only the patients who developed a significant leukopenia (No. 1, 3 , and 4) had a febrile reaction. Some product released from sequestered leukocytes may be responsible for the symptomatology observed, as has been hypothesized from studies with endotoxin (23) and red cells (16).

The thrombocytopenia observed in three of the four subjects is of particular interest. The decrease in platelets cannot be accounted for by the small numbers of circulating $\mathrm{Cr}^{51}$-labeled platelets that were destroyed. In fact, calculations showed that in each case about 25 of the subjects' own platelets were destroyed for each of the transfused labeled platelets. Lacking the appropriate antigen, these unlabeled platelets could not have combined directly with antibody but presumably were affected by small numbers of sensitized platelets in their vicinity or by some product of the platelet antigen-antibody reaction. Blood coagulation initiated by released platelet thromboplastin may also have played a role, but such "nonspecific" platelet sequestration has been shown to occur in association with other types of antigen-antibody reactions in vivo $(24,25)$. This has not been demonstrated previously for a platelet immune system. It has been suggested, however, that the rare but dramatic syndrome of posttransfusion purpura, which occurs about 7 days after transfusion of blood containing "incompatible" platelets, is brought about by a similar mechanism (1). Other thrombocytopenic disorders may be a secondary effect of in vivo antigen-antibody reactions.

Idiopathic thrombocytopenic purpura. Numerous studies have been made of platelet lifespan in ITP. Cohen, Gardner, and Barnett (7) have shown that there are at least two varieties of the disease, one caused by decreased platelet production and one caused by increased platelet destruction. In the latter type, because of temporary sequestration of $\mathrm{Cr}^{51}$-labeled platelets, it has been impossible to determine the half-disappearance time of transfused platelets when its value is less than 24 hours. For this reason, little is known of the kinetics of platelet survival in the thrombocytopenic phase of ITP or the mechanism by which adrenocortical steroids first benefit the patient. From the preliminary studies described, eliminating temporary sequestration of transfused platelets seemingly allows more effective study of these aspects of the disease.

To calculate the rate of platelet production in our patient with acute ITP would be hazardous because of uncertainty in his exact platelet level and because transfused platelets may have been utilized for hemostasis, thus shortening their lifespan. In the patient with chronic ITP, the halfdisappearance time of transfused platelets was initially 7 hours (Figure 7). The platelet level of 14,500 per $\mathrm{mm}^{3}$ did not vary significantly 
over a 3-day period of observation, indicating that she was producing platelets at the rate of 1,470 per $\mathrm{mm}^{3}$ per hour, close to the rate of 1,380 expected in a normal person (based on a platelet level of 250,000 per $\mathrm{mm}^{3}$ and an 8-day lifespan). Initially then, production was normal, but platelets were being destroyed at 19 times the usual rate. After corticosteroid therapy, the half-disappearance time of transfused platelets was doubled to 14 hours, but the platelet level rose to 60,000 . If the only effect of steroid therapy had been to prolong the half-disappearance time, a twofold increase in the platelet level would be expected. The observed fourfold increase suggests that steroids may also have induced an increase in platelet production or a mobilization of platelets from some depot. It appears that in the subject who had a normal platelet survival time the rapid beneficial response to steroid therapy was chiefly owing to enhancement of thrombopoiesis. Implicit in the above calculations is the assumption that transfused platelets were destroyed at the same rate as the subject's own platelets. If the patient has never been transfused and is not actively bleeding, this is probably true. Isoimmunity may have played a role in the second experiment in chronic ITP. We have never seen isoimmunity occur after only one transfusion, however, and it is rare in the experience of others $(7,8,26)$.

Generally, it has been assumed that the spleen represents an important site of platelet sequestration in chronic ITP because of the clinical remissions that follow splenectomy. Najean, Larrieu, and Bernard determined radioactivity in a spleen removed from a patient with ITP who had $\mathrm{Cr}^{51}$-labeled platelets transfused 4 days previously and found most of the radioactivity could be recovered there (27). Other workers have suggested the lungs (28) or some site other than the spleen (29) as repositories for platelets in this disease. The spleen was clearly the chief site of localization in the study described in Figure 7. Possibly, ITP will prove to be a disease of even greater variety than has been suspected. The technique of organ scanning after transfusion of citrate platelets may aid the decision for or against splenectomy in these patients.

Congestive splenomegaly. The normal platelet survival curves observed in two patients with congestive splenomegaly and thrombocytopenia are in accord with the observations of others (7) and support the concept that the platelet disorder in this disease is primarily one of suppressed production. The organ scanning data were difficult to interpret because of a high initial uptake of $\mathrm{Cr}^{51}$ by the spleen but are consistent with this hypothesis. The low recovery of platelets transfused to these two subjects suggests the additional possibility that the enlarged spleen or some plasma factor caused platelets affected by the $\mathrm{Cr}^{51}$-labeling procedure to be more readily destroyed than in the normal state. On several occasions, we have observed patients with alcoholic cirrhosis and thrombocytopenic purpura to undergo a sharp increase in platelet levels and cessation of purpura simply on abstaining from alcohol and ingesting a normal diet (30). Thus, toxic and nutritional factors may be the cause of thrombocytopenia as well as anemia (31-33) in such patients.

\section{Summary}

Studies were made of the sequestration of $\mathrm{Cr}^{51}$ labeled human platelets after reaction in vivo with various amounts of isoantibody. After combination with relatively small amounts of isoantibody, platelets were slowly destroyed in the spleen, whereas, with larger quantities, they were quickly destroyed in the liver. These patterns of sequestration could be induced by concentrations of antibody undetectable by in vitro serologic techniques. The destruction of platelets by platelet isoantibodies was accompanied by leukopenia and typical pyrogenic reactions.

In one case of chronic idiopathic thrombocytopenic purpura (ITP), transfused platelets were slowly destroyed in the spleen. Adrenocorticosteroids appeared to increase the circulating platelet level both by increasing platelet production and prolonging platelet survival. In a second patient, platelet survival was normal, and no organ accumulation of $\mathrm{Cr}^{51}$-labeled platelets occurred. In acute, severe ITP transfused platelets were rapidly destroyed in the liver.

It appears to be generally true that platelets mildly damaged by immunologic or other means are destroyed in the spleen but severely damaged cells are removed from the circulation by the liver. 
The use of $\mathrm{Cr}^{51}$-labeled "citrate platelets" followed by body surface scanning may permit more accurate characterization of the thrombocytopenic disorders and aid in the selection of patients for splenectomy.

\section{Addendum}

Since this manuscript was prepared, Najean and associates (Blood 1963, 22, 718), using $\mathrm{Cr}^{51}$-labeled EDTA platelets and body surface scanning, suggested that splenic uptake of $\mathrm{Cr}^{51}$ could be used to predict the results of splenectomy in idiopathic thrombocytopenic purpura. Castaldi and Firkin (Aust. Ann. Med. 1963, 12, 333) in a similar study were less optimistic in this respect.

\section{Acknowledgments}

The authors are indebted to Drs. William C. Moloney and Jane F. Desforges and to the Tufts Hematology Group at the Boston City Hospital for permission to study some of the patients described. The technical assistance of Mrs. Una F. Tuck is gratefully acknowledged.

\section{References}

1. Shulman, N. R., R. H. Aster, A. Leitner, and M. C. Hiller. Immunoreactions involving platelets. V. Post-transfusion purpura due to a complementfixing antibody against a genetically controlled platelet antigen. A proposed mechanism for thrombocytopenia and its relevance in "autoimmunity." J. clin. Invest. 1961, 40, 1597.

2. Shulman, N. R., R. H. Aster, H. A. Pearson, and M. C. Hiller. Immunoreactions involving platelets. VI. Reactions of maternal isoantibodies responsible for neonatal purpura. Differentiation of a second platelet antigen system. J. clin. Invest. 1962, 41, 1059.

3. Aster, R. H., R. H. Levin, H. E. Cooper, E. J. Friereich, E. E. Morse, and D. L. Singer. Correlation of complement-fixing antibodies with platelet survival in thrombocytopenic patients. Clin. Res. 1962, 10, 195.

4. Aster, R. H., and J. H. Jandl. Sequestration of human platelets. I. Methods. J. clin. Invest. 1964, 43, 843.

5. Jandl, J. H., M. S. Greenberg, R. H. Yonemoto, and and W. B. Castle. Clinical determination of the sites of red cell sequestration in hemolytic anemias. J. clin. Invest. 1956, 35, 842.

6. Brecher, G., and E. P. Cronkite. Morphology and enumeration of human blood platelets. J. appl. Physiol. 1950, 3, 365.

7. Cohen, P., F. H. Gardner, and G. O. Barnett. Reclassification of the thrombocytopenias by the $\mathrm{Cr}^{51}$ labeling method for measuring platelet life span. New Engl. J. Med. 1961, 264, 1284.
8. Baldini, M., N. Costea, and S. Ebbe. Studies on the antigenic structure of blood platelets in Proc. Eighth Cong. Europ. Soc. Haemat., Vienna, 1961. Basel, Karger, 1962, p. 378.

9. Stefanini, M., W. Dameshek, and E. Adelson. Platelets: VII. Shortened "platelet survival time" and development of platelet agglutinins following multiple platelet transfusions. Proc. Soc. exp. Biol. (N. Y.) 1952, 80, 230.

10. Aster, R. H., H. E. Cooper, and D. L. Singer. A simplified complement fixation test for the detection of platelet antibodies in human serum. J. Lab. clin. Med. 1964, 63, 161.

11. Aster, R. H., N. R. Shulman, H. A. Pearson, and M. C. Hiller. A complement-fixing antibody against a specific platelet antigen responsible for amegakaryocytic neonatal purpura. Clin. Res. 1961, 9, 157.

12. Aster, R. H. Unpublished observations.

13. Jandl, J. H., A. R. Jones, and W. B. Castle. The destruction of red cells by antibodies in man. I. Observations on the sequestration and lysis of red cells altered by immune mechanisms. J. clin. Invest. 1957, 36, 1428.

14. Cutbush, M., and P. L. Mollison. Relation between characteristics of blood-group antibodies in vitro and associated patterns of red-cell destruction in vivo. Brit. J. Haemat. 1958, 4, 115.

15. Harrington, W. J., C. C. Sprague, V. Minnich, C. V. Moore, R. C. Aulvin, and R. Dubach. Immunologic mechanisms in idiopathic and neonatal thrombocytopenic purpura. Ann. intern. Med. 1953, 38, 433.

16. Jandl, J. H., and A. S. Tomlinson. The destruction of red cells by antibodies in man. II. Pyrogenic, leukocytic and dermal responses to immune hemolysis. J. clin. Invest. 1958, 37, 1202.

17. De Almeida, J. O. Isofixation curves as a method of standardizing quantitative complement-fixation tests. J. Immunol. 1956, 76, 259.

18. Rapoport, M. M., and L. Graf. Immunochemical analysis based on complement fixation. Ann. N. Y. Acad. Sci. 1957, 69, 608.

19. Brittingham, T. E., and H. Chaplin, Jr. Febrile transfusion reactions caused by sensitivity to donor leukocytes and platelets. J. Amer. med. Ass. 1957, $165,819$.

20. Kevy, S. V., P. J. Schmidt, M. H. McGinniss, and W. G. Workman. Febrile, non-hemolytic transfusion reactions and the limited role of leukoagglutinins in their etiology. Transfusion (Philad.) 1962, $2,7$.

21. Dean, H. R., and R. A. Webb. The blood changes in anaphylactic shock in the dog. J. Path. Bact. 1924, 27, 65.

22. Young, L. E., D. M. Ervin, and C. L. Yuile. Hemolytic reactions produced in dogs by transfusion of incompatible dog blood and plasma. I. Serologic and hematologic aspects. Blood 1949, 4, 1218. 
23. Snell, E. S., F. Goodale, Jr., F. Wendt, and W. I. Cranston. Properties of human endogenous pyrogen. Clin. Sci. 1957, 16, 615.

24. Kopeloff, N., and L. M. Kopeloff. Blood platelets in anaphylaxis. J. Immunol. 1940, 40, 471.

25. Swisher, S. N. Nonspecific adherence of platelets and leukocytes to antibody-sensitized cells: A mechanism producing thrombocytopenia and leukopenia during incompatible transfusions (abstract). J. clin. Invest. 1956, 35, 738.

26. Freireich, E. J., A. Kliman, L. A. Gaydos, N. Mantel, and E. Frei III. Response to repeated platelet transfusion from the same donor. Ann. intern. Med. 1963, 59, 277.

27. Najean, Y., M. J. Larrieu, and J. Bernard. Survie des plaquettes marquées au radiochrome. Nouv. Rev. franc. Hémat. 1961, 1, 36.

28. Stefanini, M., J. B. Chatterjea, and W. Dameshek. Studies on platelets. V. Observations on the re- moval by the pulmonary circulation of platelets injected into patients with idiopathic thrombocytopenic purpura. J. Lab. clin. Med. 1951, 39, 865.

29. Stefanini, M., J. B. Chatterjea, W. Dameshek, C. S. Welch, and O. Swenson. Studies on platelets. - III. The absence of "selective sequestration" and destruction of platelets by the spleen in "idiopathic" thrombocytopenic purpura. Blood 1952, 7, 289.

30. Jandl, J. H., and R. H. Aster. Personal observations.

31. Jandl, J. H. The anemia of liver disease: observations on its mechanism. J. clin. Invest. 1955, 34, 390.

32. McCurdy, P. R., L. E. Pierce, and C. E. Rath. Abnormal bone marrow morphology in acute alcoholism. New Engl. J. Med. 1962, 266, 505.

33. Sullivan, L. W., and V. Herbert. Suppression of hematopoiesis by ethanol (abstract). J. clin. Invest. $1963,42,985$. 